\title{
Transcriptome analysis reveals the mechanism of improving erect-plant-type peanut yield by single-seeding precision sowing
}

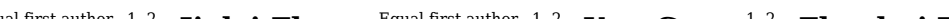
Jingjing Meng ${ }^{1,2}$, Quan Wang ${ }^{1,3}$, Shubo Wan ${ }^{\text {Corresp., }}$, Xinguo Li $^{\text {Corresp. } 1,2}{ }^{1,2}$, Jianguo Wang ${ }^{1,2}$, Feng Guo ${ }^{1,2}$,

${ }^{1}$ Biotechnology Research Center, Shandong Academy of Agricultural Sciences, Ji'nan, China

2 Scientific observation and experiment station of crop cultivation in east China, Ministry of Agriculture and Rural Affairs, Dongying, China

${ }^{3}$ College of Life Sciences, Shandong Normal University, Ji'nan, China

${ }^{4}$ Shandong Academy of Agricultural Sciences/Shandong Provincial Key Laboratory of Crop Genetic Improvement, Ecology and Physiology, Ji'nan, China Corresponding Authors: Shubo Wan, Xinguo Li

Email address: wansb@saas.ac.cn, xinguol@163.com

Background: In China, double-seed sowing (i.e., sowing two seeds per hole) has been conventionally performed towards the erect-plant-type peanuts to increase the low germination rate due to poor seed preservation conditions. However, the corresponding within-hole plant competition usually limits the subsequent plant growth and the final yield. We developed a high-yield cultivation system of single-seed precision sowing to solve this paradox, saving $20 \%$ of seeds and increasing yields by more than $10 \%$ relative to the conventional double-seed sowing. Methods: To explore the mechanisms of these two different cropping patterns in peanut yields, we conducted transcriptomic and physiological comparisons in the seeding plant leaf and root tissues between single-seed precision sowing and standard double-seed sowing treatments. Results:After assembly, each library contained an average of 43 million reads and generated a total of 523,800, 338 clean reads. After GO and KEGG pathway analysis, we found the key genes for biotic and abiotic stress showed higher expression in roots of plants grown under the single-seed precision sowing treatment, including genes encoding disease resistance, oxidationreduction, hormone related, and stress response transcription factors and signaling regulation proteins. In particular, the resveratrol synthesis genes related to stress and disease resistance appeared induced in roots under the single-seed sowing treatment. Conclusion: These data indicated that Aspergillus flavus resistance and stress tolerance in roots under single-seed precision sowing were enhanced compared with roots under the double-seed sowing treatment. This work benefits the development of underground pods and thus increasing peanut yields. 


\section{Title: Transcriptome analysis reveals the mechanism of improving}

\section{2 erect-plant-type peanut yield by single-seeding precision sowing}

3 Authors: Sha Yang ${ }^{1,2} \uparrow$, Jialei Zhang ${ }^{1,2} \uparrow$, Yun Geng ${ }^{1,2}$, Zhaohui Tang ${ }^{1,2}$, Jianguo Wang ${ }^{1,2}$, Feng

4 Guo $^{1,2}$, Jingjing Meng ${ }^{1,2}$, Quan Wang ${ }^{1,3}$, Shubo Wan ${ }^{4 *}$, Xinguo Li $i^{1,2^{*}}$

5 Co-authors Sha Yang ${ }^{1,2} \uparrow$, Jialei Zhang ${ }^{1,2 \dagger}$

6 These authors contributed equally to this work.

7 Authors' addresses

8 'Biotechnology Research Center, Shandong Academy of Agricultural Sciences, Ji'nan, 250100,

$9 \quad$ PR China

${ }^{2}$ Scientific observation and experiment station of crop cultivation in east China, Ministry of Agriculture and Rural Affairs, Dongying, 257000, PR China

${ }^{3}$ College of Life Sciences, Shandong Normal University, Ji’nan, 250014, PR China

${ }^{4}$ Shandong Academy of Agricultural Sciences/Shandong Provincial Key Laboratory of Crop

Genetic Improvement, Ecology and Physiology, Ji’nan, 250100, China

* Corresponding author Shubo $\mathrm{Wan}^{4 *}$, Xinguo $\mathrm{Li}^{1,2 *}$

${ }^{1}$ Biotechnology Research Center, Shandong Academy of Agricultural Sciences, Ji’nan, 250100, PR China

${ }^{2}$ Scientific observation and experiment station of crop cultivation in east China, Ministry of Agriculture and Rural Affairs, Dongying, 257000, PR China

${ }^{4}$ Shandong Academy of Agricultural Sciences/Shandong Provincial Key Laboratory of Crop

Genetic Improvement, Ecology and Physiology, Ji’nan, 250100, China

Tel: +86 53166659047 ;

Fax: +86 53166658156 ;

E-mail:wansb@saas.ac.cn, lixinguo@tom.com

List of all author and e-mail:

Sha Yang yangsha0904@126.com; Jialei

Zhang zhangjialei19@163.com; Yun Geng 
28 wang_jianguo2020@163.com; Feng Guo gf123456gh@126.com; Jingjing Meng

29 mengj80@163.com; Quan Wang quanwang0120@163.com; Shubo Wan wansb@saas.ac.cn;

30 Xinguo Li lixinguo@tom.com

31

32

33

34

35

36

37

38

39

40

41

42

43

44

45

46

47

48

49

50

51

52

53

54

Peer] reviewing PDF | (2020:04:48461:5:0:NEW 23 Nov 2020) 


\section{Abstract}

Background: In China, double-seed sowing (i.e., sowing two seeds per hole) has been conventionally performed towards the erect-plant-type peanuts to increase the low germination rate due to poor seed preservation conditions. However, the corresponding within-hole plant competition usually limits the subsequent plant growth and the final yield. We developed a highyield cultivation system of single-seed precision sowing to solve this paradox, saving $20 \%$ of seeds and increasing yields by more than $10 \%$ relative to the conventional double-seed sowing. Methods: To explore the mechanisms of these two different cropping patterns in peanut yields, we conducted transcriptomic and physiological comparisons in the seeding plant leaf and root tissues between single-seed precision sowing and standard double-seed sowing treatments.

Results: After assembly, each library contained an average of 43 million reads and generated a total of 523,800,338 clean reads. After GO and KEGG pathway analysis, we found the key genes for biotic and abiotic stress showed higher expression in roots of plants grown under the single-seed precision sowing treatment, including genes encoding disease resistance, oxidationreduction, hormone related, and stress response transcription factors and signaling regulation proteins. In particular, the resveratrol synthesis genes related to stress and disease resistance appeared induced in roots under the single-seed sowing treatment.

Conclusion: These data indicated that Aspergillus flavus resistance and stress tolerance in roots under single-seed precision sowing were enhanced compared with roots under the double-seed sowing treatment. This work benefits the development of underground pods and thus increasing peanut yields.

Keywords: Peanut (Arachis hypogaea L.), RNA-Seq, Stress tolerance, Resveratrol synthase, 
82

83

84

85

86

87

88

89

90

91

92

93

94

95

96

97

Yield

4

\section{Introduction}

Before the 1960s, agricultural production in China was labor-intensive due to the lack of modern farming conditions. Thus, erect-plant-type peanuts, which are easier for manual labor relative to prostrate-plant-type peanuts, were successfully bred and cultivated widely in China. Because the seed germination rate of peanut is usually unable to meet the necessary high field emergence percentage owing to uneven seed quality, conventionally, the sowing patterns with double-seed (DS) and multi-seed in one hole were widely adopted by farmers as the main planting patterns, which generally leads to two issues: a requirement of a large number of seeds and poor population quality. The total amount of peanut seeds per year is 1.5 million tons, accounting for about $10 \%$ of the country's total peanut yield. Poor population quality is mainly attributed to the increased competition under double-seed sowing, which hinders the increases of peanut yield (Zhang et al., 2020).

Our team introduced the principle of competitive exclusion, proposed the technical idea of "single-seed (SS) precision sowing of erect-plant-type peanuts, robust individual, optimized population", and created a high-yield cultivation technique of single-seed precision sowing. SS precision sowing can alleviate inter-plant competition, save $20 \%$ of seeds used for planting, and increase yields by more than $10 \%$ in common fields relative to DS precision sowing (Liang et al., 2019). However, the molecular mechanisms regulating individual development under SS are still unknown.

Crop yield is usually affected by biotic and abiotic stress. In recent years, biotic stress research has mainly focused on disease resistance in wheat, rice, maize, and soybean. It has been reported that $F h b 1$ was associated with resistance to wheat scab and has important breeding potential 
109 (Rawat et al., 2016). Two grape stilbene synthase (STS) genes, VST1 and VST2, were 110 transformed into tobacco for the first time, which improved tobacco resistance to Botrytis 111 cinerea (Hain et al., 1993). "MutRenSeq," proposed by a British research team, is an efficient 112 method to discover disease-resistant genes in crops (Steuernagel et al., 2016). These advances 113 lay a solid foundation for the further study of crop disease resistance.

114 Aflatoxin contamination caused by Aspergillus flavus is a major obstacle to the development 115 of peanuts (Fajardo et al., 1994). It was indicated that the resveratrol content could be 116 significantly induced when Aspergillus flavus infects the peanuts seeds; and resistance of peanut 117 to Aspergillus flavus infection can be conferred by increasing the content of resveratrol in peanut 118 seeds as well as the speed of its synthesis (Tian et al., 2008). Resveratrol (Res), known as 3,4,5119 trihydroxy-stilbene, was first isolated from Veratrum grandiflorum root in 1940 (Takaoka 1940). At present, Res has been identified in many different plant species, and recent research is mainly focusing on grapes (Wang et al., 2010), peanuts (Tang et al., 2010), and a few other species. Grape pericarps are the main grape tissue in which the compound is synthesized. The content of Res in peanut pericarps is higher than that in peanut kernels (Sanders et al., 2000). Accumulated of Res, a non-flavonoid polyphenol, could improve abiotic stress resistance. Although there has remained a query of the specific mechanisms of Res's anti-stress abilities, a recent report showed that Res resistance to ROS is one aspect involved in the stress-resistant processes (Zheng et al., 127 2011).

Abiotic stresses, including drought, salt, and extreme temperature, remain the most challenging frontier in the field of plant abiotic interaction research. Research on abiotic stress focuses on identifying primary sensors and essential stress-resistant genes that respond to abiotic stresses $(Z h u, 2016)$. Significant progress has been made in the past few years. The cold-tolerant rice protein COLD1 has been identified to mediate extracellular $\mathrm{Ca}^{2+}$ influx and net cytosolic $\mathrm{Ca}^{2+}$ concentration in response to chilling stress (Ma et al., 2015). The basic leucine zipper bZIP and NAC transcription factors may sense or contribute tolerance to salt stress through their interaction with corresponding proteins (Liu et al., 2016). The chloroplast is the main site of the 
136

137

138

139

140

141

142

143

144

145

146

147

148

149

150

151

152

153

154

155

156

157

158

159

160

161

162

production of reactive oxygen species (ROS), including hydrogen peroxide, superoxide anions, singlet oxygen, and hydroxyl radicals (Mignolet-Spruyt et al., 2016). Various abiotic stresses, particularly high light stress, aggravate ROS production, destroying ROS-scavenging systems and generating various secondary messengers. The protective mechanisms that scavenge ROS in plants can be divided into two categories: enzymatic (e.g., superoxide dismutase [SOD], catalase [CAT], and peroxidase [POD]) mechanisms and non-enzymatic (e.g., glutathione, mannitol, and flavonoids) mechanisms. Improving the content of these substances in plants by genetic engineering can effectively remove excessive ROS in plants and thus improve stress tolerance. Whether the yield advantage of SS precision sowing is related to the stress resistance genes needs to be studied.

With the development of high-throughput sequencing technologies, more attention is being paid to combining genomic methods such as genome sequencing with transcriptome, proteome, and metabolome analyses in order to reveal the molecular mechanism of life science research. This integrative research offers synergies that can uncover the molecular mechanism of different phenomena in crop production. The publication of reference transcripts for Arachis_duranensis (Chopra et al., 2014) facilitated a better understanding of agronomically important phenomena and genetic improvement of peanuts. This study used these techniques mentioned above to reveal the mechanism attributing to the higher peanuts yield by SS precision sowing.

\section{Materials \& Methods}

\section{Plant materials and growth conditions}

Peanut (Arachis hypogaea L.) cultivar 'Huayu 22' (provided by the Shandong Peanut Research Institute, China), a large-grain peanut cultivar, was used in this study. Peanut seeds were cultivated under field pot conditions at the Shandong Academy of Agricultural Sciences Station $\left(117^{\circ} 5^{\prime} \mathrm{E}, 36^{\circ} 43^{\prime} \mathrm{N}\right)$, Ji'nan, China. The soil is sandy loam, containing $1.1 \%$ organic matter (W/W), $82.7 \mathrm{mg} \mathrm{kg}^{-1}$ alkali-hydrolyzed nitrogen, $36.2 \mathrm{mg} \mathrm{kg}^{-1}$ available phosphorus, $94.5 \mathrm{mg} \mathrm{kg}^{-}$ 1 available potassium, and $14.9 \mathrm{~g} \mathrm{~kg}^{-1}$ exchangeable calcium. The single-seed (SS) sowing treatment consisted of 237,000 holes per hectare with 1 grain per hole, while the double-seed 
163

164

165

166

167

168

169

170

171

172

173

174

175

176

177

178

179

180

181

182

183

184

185

186

187

188

189

(DS) sowing consisted of 138,000 holes per hectare with two grains per hole as the control; the distances between two adjacent holes were $10.5 \mathrm{~cm}$ and $18 \mathrm{~cm}$ in the SS and DS treatments, respectively. Three biological replicates were used in this study. The mixed tissues were used in transcriptome sequencing.

\section{RNA extraction and cDNA library construction}

When the peanut plants reached the seedling stage, the top third leaf and the whole root systems were collected for RNA extraction and library construction. Total RNA was isolated from the leaves and roots respectively, using the total RNAiso Reagent (TaKaRa, Dalian, China) according to the manufacturer's instructions. The quality and purity of RNA samples were detected using the Agilent 2100 Bioanalyzer platform and Agilent RNA 6000 Nano Kit (Agilent, Santa Clara, CA, USA). Qualified RNA samples were used to isolate mRNA with the oligo (dT) method, and the mRNAs were fragmented. Then, first-strand cDNA and second strand cDNA were synthesized. Next, the purified cDNA fragments were linked with adapters, and sequencing was performed by a commercial service provider (BGI Tech, Shenzhen, China). The sequenced reads that contained adaptor sequences, low-quality bases, and high contents of unknown bases (i.e., $\mathrm{N}$ calls) were removed before downstream analyses. After read filtering, clean reads were mapped to the Arachis_duranensis reference genome (BioSample: SAMN02982871, BioProject: PRJNA258023) using Hierarchical Indexing for Spliced Alignment of Transcripts (HISAT)._The sequence data can be located from https://www.ncbi.nlm.nih.gov/assembly/GCF_000817695.2/.Then, Genome Analysis Toolkit (GATK) was used to call single SNPs and INDELs (McKenna et al., 2010), and RNA-Seq by Expectation-Maximization (RSEM) was used to calculate gene expression levels for each sample, which were then determined using the fragments per kb per million (FPKM) mapped fragments method developed by Li et al. (2011) (Li et al., 2011). DEGseq algorithms were used to assess the DEGs. Based on the gene expression level, DEG was identified between samples or groups. Gene Ontology (GO) was performed classification and Kyoto Encyclopedia of Genes and 
190

191

192

193

194

195

196

197

198

199

200

201

202

203

204

205

206

207

208

209

210

211

212

213

214

215

216

Genomes (KEGG) pathway classification based on these DEGs. The GO framework includes three ontologies: molecular biological function, cellular component, and biological process. KEGG is a database resource for understanding high-level functions and utilities of the biological system, such as the cell, the organism, and the ecosystem, from molecular-level information, especially large-scale molecular datasets generated by genome sequencing and other high-throughput experimental technologies. Co-expression analysis was performed using the WGCNA R package according to the methods detailed by previously published studies (Gao et al., 2018; Song et al., 2018).

\section{Yield composition per plant and measurement}

At maturity, ten representative plants were selected to investigate the number of pods, full pods, and double kernels, as well as pod weight per plant. The pods and plants were dried to constant weights, and the economic coefficient was calculated as follows: economic coefficient = pod dry weight / (plant dry weight + pod dry weight).

\section{Antioxidant enzyme activities and root activity}

Leaf tissues $(0.5 \mathrm{~g})$ were ground up with phosphate buffer $(\mathrm{pH} 7.8)$ containing $0.1 \mathrm{mM}$ EDTA and $1 \%\left(\mathrm{~g} \mathrm{ml}^{-1}\right)$ PVP and centrifuged at $4{ }^{\circ} \mathrm{C}$ for $10 \mathrm{~min}$. Then, the supernatants were used as enzyme extracts. The nitroblue tetrazole (NBT) method was used to detect SOD activity. The absorbance value was determined at a $560 \mathrm{~mm}$ wavelength after the reaction; $50 \%$ inhibition of NBT reduction was regarded as the enzyme activity unit (U), expressed as $\mathrm{U}^{-1} \mathrm{FW}$. The guaiacol method was used to determine the POD activity; the increase in $\mathrm{OD}_{470}$ per min was used as the enzyme activity unit (U), expressed as $\Delta 470 \mathrm{~g}^{-1}$. CAT activity was also determined by the guaiacol method. The decreased in $\mathrm{OD}_{240}$ per min was used as the unit of enzyme activity (U) and expressed as $\mathrm{mg} \cdot \mathrm{g}^{-1} \cdot \mathrm{min}^{-1}$ (Giannopolitis et al., 1977; Aebi et al., 1984; Jimenez et al., 1977). Root activity was measured by the TTC method; $0.5 \mathrm{~g}$ of root tip samples were revolved in a mixture of $0.4 \%$ TTC and phosphoric acid buffer solution and kept in the dark for $1-3 \mathrm{~h}$ at $37^{\circ} \mathrm{C}$. 
217 After that, $2 \mathrm{ml}$ of $1 \mathrm{~mol} \mathrm{~L}^{-1}$ sulfuric acid was added to stop the reaction. Simultaneously, a blank 218 experiment was conducted. The root samples and sulfuric acid were added first, and 10 min later, 219 the other reagents were added. The operation was the same as that described above. The roots 220 were removed, dried, and ground together with $3-4 \mathrm{ml}$ of quartz sand and ethyl acetate in a

221

222

223

mortar. The supernatants were transferred to a test tube, and the residue was washed with a small amount of ethyl acetate two or three times, with all ethyl acetate collected into the test tube. Finally, ethyl acetate was added to bring the total volume up to $10 \mathrm{ml}$. Blank experiments were used as the control, and the OD value of the reaction solution was measured at $\lambda=485 \mathrm{~nm}$.

\section{qRT-PCR verification analysis}

Leaves and roots of plants grown under the SS and DS treatments, as in the RNA-seq experiment, were selected randomly and analyzed using qRT-PCR. The qRT-PCR amplification instrument (ABI 7500 fast; Applied Biosystems, Foster City, CA, USA) was used to amplify the related genes with SYBR Premix Ex Taq ${ }^{\mathrm{TM}}(\mathrm{TaKaRa})$ following the manufacturer's instructions (Yang

et al., 2013). The relative gene expression was calculated using the $2^{-\triangle \triangle \mathrm{CT}}$ method described by Livak and Schmittgen (2001) (Livak \& Schmittgen, 2011). For $\log _{2}$-transformed FPKM values, each selected gene's maximum expression level was considered to be 100 , and the expression levels of the other genes were transformed accordingly (Zhang et al., 2016).

\section{Determination of resveratrol content}

The Res extraction processes were carried out according to the method detailed by Tang et al. (Tang et al., 2010) with minor modification; $0.25 \mathrm{~g}$ of leaves or roots were collected and ground into a fine powder, which was dissolved with $20 \mathrm{ml}$ of $95 \%$ ethanol in a flask and oscillated at room temperature overnight. After centrifugation at $8000 \mathrm{rpm}$ for $10 \mathrm{~min}$, the supernatants were subjected to HPLC analysis. The extracts were filtered through a $0.45-\mu \mathrm{m}$ membrane and then tested with the Rigol L3000 HPLC system (Rigol Technologies, Beaverton, OR, USA) using a Kromasil C18 reversed phase column (4 $\mu \mathrm{m}, 250 \mathrm{~mm} \times 4.6 \mathrm{~mm}$; Kromasil, Bohus, Sweden) at 
244 room temperature. Mobile phase A consisted of $0.1 \%$ phosphoric acid $-\mathrm{H}_{2} \mathrm{O}$, while phase B 245 consisted of acetonitrile $\left(\mathrm{A}: \mathrm{B}=70: 30\right.$, flow rate $\left.1 \mathrm{ml} \mathrm{min}^{-1}\right)$; the determination wavelength was $246280 \mathrm{~nm}$.

\section{Results}

\section{Transcriptome sequencing and gene analysis}

249 The peanut plants grown under different planting patterns differed little in their above-ground portions, while great differences were observed between their root systems. The basis of high

251

252

253

254

255

256

257

258

259

260

261

262

263

264

265

266

267

268

269

270 and stable crop yields is high biological yields, which depends largely on the development of root systems. The capillary lateral root is the main type of root exhibited under SS. Thus, the capillary lateral root length and its proportion within the root system were promoted accordingly, thereby increasing the total root length and root absorption area of peanut plants. This phenomenon was verified by the mean absorbing area and dry weight of root tissue observed under SS (Fig. 1).

Twelve cDNA libraries were constructed from leaf and root tissues of peanut plants grown under SS and DS sowing treatments. All 12 samples were sequenced on the Illumina HiSeq Platform in total, generating about $6.55 \mathrm{~Gb}$ per sample. The reads that contained adaptor sequences of low-quality or had high contents of unknown base (i.e., N) calls were removed, resulting in a total of 523,800,338 clean reads that were acquired, with an average of 43 million reads per libraries (Table 1). Approximately $77 \%$ and $80 \%$ of reads from leaf and root tissues were mapped to the reference genome, and 36,778 genes were identified, of which 34,529 are known genes and 2,322 are novel genes. The RNA-seq sequencing data for the present work has been uploaded in NCBI Sequence Read Archive under BioProjects, PRJNA497502 (SRA: SRP166140).

Single nucleotide polymorphisms (SNP), which include transitions or transversions of single bases, refer to the difference of a single nucleotide (A, T, C, or G) between homologous DNA sequences, a key element of the diversity of genomes among species or individuals. There were more transitions than transversions among all the samples. Among SNPs, transition, transversion, 
271 A-G, and C-T were the most abundant terms (Fig. S1). Insertion-Deletion (INDEL) refers to the

272 insertion or deletion of the small fragments (one or more, less than $50 \mathrm{bp}$ ) that occur in a sample

273 relative to the reference genome. Most INDELs occurred in exonic and intronic sequences, with

274 the proportion differing among samples (Fig. S2). Based on the results of SNP, INDEL, and gene

275 expression, our study was presented in the form of a ring diagram with Circos software (Fig. S3).

\section{Differentially expressed gene detection}

277 A total of 2,567 and 2,706 differentially expressed genes (DEGs) were identified from leaves and 278 roots, respectively. Under SS sowing treatment, 544 and 1,771 genes were expressed at a higher

279 level, while 2,023 and 935 genes were expressed at lower levels compared with the DS sowing 280 treatment in leaves and roots, respectively (Fig. 2). The expression patterns of the majority of DEGs differed between roots and leaves. For example, only $83(15.2 \%)$ of the 1,771 genes upregulated in roots were also up-regulated in leaves (Fig. 3). Notably, some genes in roots and leaves even showed opposite expression patterns; for example, forty DEGs were up-regulated in leaves but down-regulated in roots. However, 245 DEGs in roots and leaves had the same expression trends, including 83 up-regulated DEGs and 162 down-regulated DEGs (Fig. 3).

qRT-PCR verification of RNA-Seq results

To verify the RNA-seq data, quantitative real-time PCR (qRT-PCR) was used to test the expression of 24 genes with different functional assignments (Fig. 4a, 4b). Among them, six and five genes were up-regulated under the SS sowing treatment in leaves and roots, respectively. UDP-glycosyltransferase, chalcone synthase, and GPI mannosyltransferase were each encoded by one of these genes, while two genes encoding hypothetical proteins were also used for qRTPCR detection. The other eleven selected genes were downregulated both in roots and leaves. These genes included one phospholipase, one seed linoleate 9S-lipoxygenase, one zinc finger SWIM domain-containing protein, and one pectinesterase. The RNA-seq data were consistent with the qRT-PCR results for these 24 genes. The correlation between the relative expression 
$2980.9772)$ and a little lower in roots $\left(R^{2}=0.8884\right.$; Fig. $\left.4 \mathrm{c}, 4 \mathrm{~d}\right)$.

299

300

301

302

303

304

305

306

307

308

309

310

311

312

313

314

315

316

317

318

319

320

321

322

323

324

\section{Functional analysis of DEGs}

Blast2GO was used to perform Gene Ontology (GO) classification and functional enrichment (Conesa et al., 2005) on the identified DEGs. The sequences were categorized into 45 functional groups according to sequence homology. The main categories of biological process, molecular function, and cellular component were visualized employing WEGO (Fig. 5). In the biological process category, "metabolic process", "single-organism process", and "cellular process" terms were enriched, suggesting the metabolic activity is higher under SS sowing. In the cellular component category, "cell," "cell part," and "membrane" were the most abundant terms. The most enriched category was molecular function, with enrichment for "binding," "catalytic activity," and "transporter activity" in particular, suggesting a high level of metabolic activity changes under SS sowing (Fig. 5).

KEGG pathway classification was performed to acquire biological information for understanding the regulatory networks and molecular mechanisms associated with the SS sowing treatment. DEGs were mainly enriched in the MAPK signaling pathway, glycerolipid metabolism, phenylalanine metabolism, sphingolipid metabolism, isoflavonoid biosynthesis, flavonoid biosynthesis, and tryptophan metabolism in leaves. Meanwhile, phenylpropanoid biosynthesis, biosynthesis of secondary metabolites, MAPK signaling pathway, flavonoid biosynthesis, zeatin biosynthesis, and flavone and flavonol biosynthesis were mainly enriched among DEGs in roots (Fig. S4). Interestingly, all of these pathways participated in biosynthesis associated with particular metabolic processes, suggesting that these processes were activated. Weighted gene correlation network analysis (WGCNA) is a systematic biological method for describing gene association pattern among different samples. Information about nearly 10,000 genes, corresponding to the genes with the greatest changes in expression, was used to identify gene sets of interest and analyze associations with phenotypes. In our study, the genes from all the samples were divided into modules for analysis, with twelve colors representing each of the 
325

326

327

328

329

330

331

332

333

334

335

336

337

338

339

340

341

342

343

344

345

346

347

348

349

350

351

different modules in Fig. 6.

\section{Changes of resveratrol synthesis related pathway between cultivation techniques}

In our study, a total of 20 resveratrol synthesis-related genes were dramatically induced, while no resveratrol synthesis-related genes were down-regulated in the roots under the SS sowing treatment. In contrast, a total of 10 DEGs related to resveratrol synthesis were down-regulated in the leaves (Table 2). We also measured the resveratrol content, and the results were in agreement with the gene expression levels in leaf and root tissues (Fig. 7). Accordingly, compared with the roots of plants grown under DS sowing, the higher resveratrol content under SS precision sowing appeared to support the initiation of the defense response against toxins produced by Aspergillus flavus; pathogenesis-related proteins, transcription factors (e.g., WRKY, bZIP, ERF), and 4CL were up-regulated (Table 3).

\section{Key genes related to stress tolerance under SS}

Transcription factors (TFs), which bind to the cis-elements upstream of promoters, have been reported to orchestrate abiotic stress responses (Joshi et al., 2016). Under the SS precision sowing treatment, the higher expression of these TF genes (WRKY, MYB, bZIP, ERF) may improve stress tolerance compared with that observed in the DS treatment. In plants, redox processes also play an important role in stress tolerance. Several oxidoreductase genes encoding ascorbate oxidase, glutaredoxin, and cytochrome P450 monooxygenases (CYP) exhibited increased expression in roots under SS relative to DS. Specifically, compared with DS, about thirty genes encoding CYP were up-regulated under SS (Table 3). In addition, four genes encoding L-ascorbate oxidase homologs, which take part in ascorbate recycling, were enriched in the SS sowing treatment. In plants, ascorbate contributes to improving tolerance against various stresses by regulating the levels of cellular $\mathrm{H}_{2} \mathrm{O}_{2}$ (Ishikawa et al., 2008). Higher enzyme activities of POD, SOD, and CAT were also observed in roots under SS compared to that under DS (Table S1). Four genes encoding glutaredoxin, which might promote reducing disulfide 
352 bridges, were slightly induced under SS compared with DS (Table 3).

353

354

355

356

357

358

359

360

361

362

363

364

365

366

367

368

369

370

371

372

373

374

375

376

377

378

\section{Peanut pod yields and associated genes under the SS sowing pattern}

The number of pods and pod weight per plant is the direct factors affecting peanut yield. In this study, yield estimation indicated that the number of pods, full pods, and double kernels per plant under SS was higher than under DS. The theoretical pod yield of SS reached $1.225 \mathrm{~kg}$, which was $12.9 \%$ higher than that of DS (1.085) (Table 4). Hormones, such as indole-3-acetic acid (IAA), abscisic acid (ABA), gibberellin (GA), and brassinosteroid (BR), play important roles in the regulation of growth and development in plants (Tian et al., 2017). Auxin response factor (ARF), a transcription factor involved in auxin and regulating plant root growth and seed development (Salmon et al., 2008), showed increased expression levels under SS. Nine genes that participate in auxin and BR biosynthesis were abundantly expressed under SS compared with under DS (Table 3). Two lipoxygenase family genes also had increased expression levels under SS.

\section{Discussion}

In recent years, with the rapid development of sequencing technology, genomes and transcriptomes have been analyzed to explore the mechanisms mediating various problems in agricultural production. However, few studies have focused on the mechanisms by which singleseed precision sowing improves yields in erect-plant-type peanut cultivars. Aerial flowering combined with underground fruit development are special characteristics of peanut plants; thus, the growth and development of root tissues are particularly important for peanut yields. Robust roots are the basis of crop growth and high yields (Zheng et al., 2013).

Various biotic and abiotic stresses under adverse environmental conditions severely reduce global crop production and food security (Mengiste et al., 2003). Peanut is one of the most susceptible crops to Aspergillus flavus. Abiotic stresses such as salinity, heat, mechanical damage, and drought seriously affect peanut crops' growth and development (Amin et al., 2019). 
379 In our study, families of TFs, including Zinc finger protein, MYB, bZIP, ERF, and WRKY, were 380 increased in the SS sowing treatment compared with the DS sowing treatment. Many studies 381 have identified these TFs as being involved in abiotic stress responses (Wei et al., 2017; Wang et 382 al., 2016; Banerjee et al., 2015). In addition, secondary messengers, like $\mathrm{Ca}^{2+}$ and ROS, trigger 383 signaling proteins downstream, such as calcium-dependent protein kinases (CDPKs), calmodulin 384 (CaM), calcineurin-B-like proteins (CBLs), mitogen-activated protein kinase (MAPK) cascades, 385 and ROS-modulated protein kinases (PKs), and these can also respond to numerous plant 386 387 388 development and environmental challenges (Yang et al., 2019). Genes encoded calcium-binding protein CML, calcium-binding protein KIC-like, and MAPKKK, which is part of the $\mathrm{Ca}^{2+}$ signaling pathway, were expressed at higher levels under SS (Table 3). Genes involved in the MAPK cascade include interlinked MAPK, MAPKK, and MAPKKK, and such cascades play important roles in signal transduction of plant hormones, biotic stresses, and abiotic stresses (Wang et al., 2017).

Resveratrol (Res), an important phytoalexin, can be induced by pathogenic bacteria or other 393 stimuli and has strong bactericidal and defensive activity in plants. So far, the heterogeneous transformation of Res genes from rice, barley, wheat, tomato, tobacco, and other crops has been reported. All these transgenic crops exhibit improved resistance to diseases such as rice blast (Stark et al., 1997), powdery mildew in barley and wheat (Fettig et al., 1999), and gray mold in tomato and tobacco (Hain et al., 1990). The rate of Res induction in peanut seeds has also been linked to resistance to Aspergillus flavus infection (Fajardo et al., 1994). Res is synthesized by the phenylalanine metabolic pathway in plants, which includes four key enzymes: cinnamate-4hydroxylase $(\mathrm{C} 4 \mathrm{H})$, phenylalanine ammonia lyase (PAL), 4-coumarate-CoA ligase (4CL), and STS. STS genes can be divided into two types, one of which is resveratrol synthase (RS), which synthesizes Res using malonyl-CoA and coumaric acid-CoA as substrates, and this type mainly exists in Arachis hypogaea and Vitis vinifera. The other is pinosylvin synthase (PS), which utilizes malonyl-CoA and cinnamyl-CoA as substrates, and has mainly been identified in Pinus 405 sylvestris and Pinus strobus. RS is the major rate-limiting enzyme for resveratrol synthesis, 
406

407

408

409

410

411

412

413

414

415

416

417

418

419

420

421

422

423

424

425

426

427

428

429

430

431

432

which is reported to participate in ROS resistance and is a key element in stress resistance processes (Chang et al., 2011). Endogenous or exogenous Res can also reduce ROS content in plants (Zheng et al., 2015). These Res's effects are likely to promote disease resistance and antioxidant ability in peanut roots, thus supporting high-quality harvests and high yields of peanuts.

In this study, high activities of the enzymes POD, SOD, and CAT in the ROS scavenging pathway were observed in roots of plants grown under SS (Table S1). SOD, as a key element of enzymatic defense systems, catalyzes the disproportionation of radicals $\mathrm{O}_{2}{ }^{-}$to $\mathrm{O}_{2}$ and $\mathrm{H}_{2} \mathrm{O}_{2}$, the latter of which can then be scavenged by POD or CAT. The elevated activity of antioxidant enzymes can be regarded as an effective mechanism for resisting oxidative stress. The balance of the redox state of plant cells contributes to improving plant resistance to abiotic stress (Duan et al., 2012). It has been reported that overexpression of glutaredoxin in tomatoes can confer drought, oxidation, and salt resistance (Guo et al., 2010). These results suggested oxidoreductases are involved in protecting plants from various abiotic stresses through maintaining oxidation-reduction homeostasis and scavenging surplus ROS. CYP catalyzes the biological oxidation of various substrates through molecular oxygen activation and acts as a pivotal part of stress responses and metabolic processes, exhibited as differential transcription levels under SS and DS treatments (Xiong et al., 2017).

Based on previous studies, auxin and BR are known to play important roles in root development. Still, the root tip phenotypes of auxin mutants differ from those of BR mutants (González-García et al., 2011), indicating that the auxin and BR pathways acting on the root are not the same. In Arabidopsis, the BR encoding gene AtDWF4 was found to regulate leaf growth by promoting cell expansion (Hur et al., 2015). Its overexpression in Brassica napus can increase seed production (Sahni et al., 2016). Many unigenes encoding auxins and BR biosynthesis-related genes are expressed at a high level under SS. Lipoxygenase, which exhibited different expression levels between SS and DS treatments, is an enzyme that is important to the synthesis of jasmonic acid, which plays a vital role in stored lipid migration during seed 
433

434

435

436

437

438

439

440

441

442

443

444

445

446

447

448

449

450

451

452

453

454

455

456

457

458

459

germination (Rahimi et al., 2016). The up-regulated expression of lipoxygenases under SS is likely to provide sufficient nutrients essential for pod development. ABA also plays crucial roles in seed dormancy and development (Chauffour et al., 2019). These results strongly suggested that the biotic and abiotic stress tolerance in roots of peanut plants grown from single-seed precision sowing were enhanced compared with those under the double-seed sowing treatment. These mechanisms may explain the higher yields achieved under SS precision sowing based on patterns at the transcriptional level.

\section{Conclusions}

This study performed a comparative transcriptomic analysis of peanut leaves and roots between precision SS sowing and standard DS sowing treatments. Genes involved in resveratrol synthesis were found to be dramatically induced in roots under the SS treatment. Accordingly, a higher content of resveratrol was detected under SS precision sowing. In addition, genes involved in calcium signaling transduction and phytohormone metabolism were identified to be differentially expressed in SS and DS roots. Redox process genes and transcription factors, including WRKY, MYB, MADS-box, and zinc finger proteins, were up-regulated in roots of plants grown under the SS precision sowing treatment. These genes participate in resistance to biotic and abiotic stresses, thus protecting plants from disease and drought, salinity, and chilling stress. These results may inform breeding to enhance roots' activity and growth, providing a basis for achieving higher yields in peanuts. To the best of our knowledge, we have provided a novel mechanism by which SS precision sowing improves erect-plant-type peanut yields.

\section{Funding}

The following grants supported this work: National Key R\&D Program of China (2018YFD1000900); Major Basic Research Project of Natural Science Foundation of Shandong Province (2018GHZ007); Science and Technology Innovation Project of Shandong Academy of Agricultural Sciences (CXGC2018D04); Agricultural scientific and technological innovation project of Shandong Academy of Agricultural Sciences (CXGC2018E13).

\section{List of abbreviations}


460

461

462

463

464

465

466

467

468

469

470

471

472

473

474

475

476

477

478

479

480

481

482

483

484

485

486

ABA, Abscisic acid; BR, Brassinosteroid; ARF, Auxin response factor; CAT, Catalase; CBLs, Calcineurin-B-like proteins; CDPKs, Calcium-dependent protein kinases; C4H, Cinnamate-4hydroxylase; CIPK, CBL-interacting protein kinase; 4CL, 4-coumarate-CoA ligase; CYP, Cytochrome P450; DEGs, Differentially expressed genes; FPKM, Fragments per kb per million; GA, Gibberellin; GATK, Genome analysis toolkit; GO, Gene ontology; HISAT, Hierarchical indexing for spliced alignment of transcripts; IAA, Indole-3-acetic acid; INDEL, InsertionDeletion; MAPK, Mitogen-activated protein kinase; NBT, Nitroblue tetrazole; PAL, Phenylalanine ammonia-lyase; POD, Peroxidase; PS, Pinosylvin synthase; qRT-PCR, Quantitative real-time PCR; Res, Resveratrol; RSEM, RNA-Seq by expectation-maximization; SNP, Single nucleotide polymorphisms; SOD, Superoxide dismutase; STS, Stilbene synthase; WGCNA, Weighted gene correlation network analysis.

\section{Competing Interests}

The authors declare that they have no competing interests.

\section{Authors' Contributions}

SW and XL designed the study, SY and JZ carried out most of the experiments and data analysis and wrote part of the manuscript. YG wrote part of the manuscript and finalized the figures and tables. ZT, FG, and JW performed part of the experiments. JM and QW tended the plants. All authors have read and approved the manuscript.

\section{References}

Aebi H. 1984. Catalase in vitro. Methods Enzymol 105:121-126.

Amin AB, Rathnayake KN, Yim WC, Garcia TM, Wone B, Cushman JC, Wone BWM. 2019. Crassulacean acid metabolism abiotic stress-responsive transcription factors: a potential genetic engineering approach for improving crop tolerance to abiotic stress. Frontiers in Plant Science 10:129.

Banerjee A, Roychoudhury A. 2015. WRKY proteins: signaling and regulation of expression during abiotic stress responses. The Scientific World Journal 2015:807560.

Chang X, Heene E, Qiao F, Nick P. 2011. The phytoalexin resveratrol regulates the initiation of 
hypersensitive cell death in Vitis cell. Plos One 6: e26405.

488 Chauffour F, Bailly M, Perreau F, Cueff G, Suzuki H, Collet B, Frey A, Clément G, Soubigou489 Taconnat L, Balliau T, Krieger-Liszkay A, Rajjou L, Marion-Poll A. 2019. Multi-omics 490 analysis reveals sequential roles for ABA during seed maturation. Plant $491 \quad$ Physiology pp.00338.

492 Chopra R, Burow G, Farmer A, Mudge J, Simpson CE, Burow MD. 2014. Comparisons of de 
514 Guo YS, Huang CJ, Xie Y, Song FM, Zhou XP. 2010. A tomato glutaredoxin gene SlGRX1 515 regulates plant responses to oxidative, drought and salt stresses. Planta 232:1499-1509.

516 Hain G, Reif HJ, Krause E, Langebartels R, Kindl H, Vornam B, Wiese W, Schmelzer E, 517 Schreier PH, StÖcker RH, Stenzel K. 1993. Disease resistance results from foreign 518 phytoalexin expression in a novel plant. Nature 361:153-156.

519 Hain R, Bieseler B, Kindl H. 1990. Expression of a stilbene gene in Nicotiana tabacum results in $520 \quad$ synthesis of the phytoalexin resveratrol. Plant Molecular Biology 15:325-335.

521 Hur YS, Um JH, Kim S, Kim K, Park HJ, Lim JS, Kim WY, Jun SE, Yoon EK, Lim J, Ohme522 Takagi M, Kim D, Park J, Kim GT, Cheon CI. 2015. Arabidopsis thaliana homeobox 12 523 
541 Livak KJ, Schmittgen TD. 2001. Analysis of relative gene expression data using real-time 542 quantitative PCR and the 2(-Delta Delta C(T)) Method. Methods 25(4):402-408.

543 Ma Y, Dai X, Xu Y, Luo W, Zheng X, Zeng D, Pan Y, Lin X, Liu H, Zhang D, Xiao J, Guo X, 544 Xu S, Niu Y, Jin J, Zhang H, Xu X, Li L, Wang W, Qian Q, Ge S, Chong K. 2015. COLD1 $545 \quad$ confers chilling tolerance in rice. Cell 160(6):1209-1221.

546 McKenna A, Hanna M, Banks E, Sivachenko A, Cibulskis K, Kernytsky A, Garimella K, 547 Altshuler D, Gabriel S, Daly M, DePristo MA. 2010. The genome analysis toolkit: a

Mengiste MapReduce framework for analyzing next-generation DNA sequencing data. Genome Research 20:1297-1303.

551

552

553

554

555

556

557

558

559

560

561

562

563

564

565

566

567
T, Chen

X, Salmeron
J, Dietrich

R. 2003. The BOTRYTIS SUSCEPTIBLE1 gene encodes an R2R3MYB transcription factor protein th at is required for biotic and abiotic stress responses in Arabidopsis. The Plant Cell 15(11):2551-2565.

Mignolet-Spruyt L, Xu E, Idanheimo N, Hoeberichts FA, Muhlenbock P, Brosche M, Van Breusegem F, Kangasjarvi J. 2016. Spreading the news: subcellular and organellar reactive oxygen species production and signalling. Journal of Experimental Botany 67:3831-3844.

Rahimi S, Kim YJ, Sukweenadhi J, Zhang D, Yang DC. 2016. PgLOX6 encoding a lipoxygenase contributes to jasmonic acid biosynthesis and ginsenoside production in Panax ginseng. Journal of Experimental Botany 67(21):6007-6019.

Rawat N, Pumphrey MO, Liu S, Zhang X, Tiwari VK, Ando K, Trick HN, Bockus WW, Akhunov E, Anderson JA, Gill BS. 2016. Wheat Fhbl encodes a chimeric lectin with agglutinin domains and a pore-forming toxin-like domain conferring resistance to Fusarium head blight. Nature Genetics 48 (12):1576-1580.

Sahni S, Prasad BD, Liu Q, Grbic V, Sharpe A, Singh SP, Krishna P. 2016. Overexpression of the brassinosteroid biosynthetic gene DWF4 in Brassica napus simultaneously increases seed yield and stress tolerance. Scientific Reports 6:28298.

Salmon J, Ramos J, Callis J. 2008. Degradation of the auxin response factor ARF1. The Plant 
Journal 54:118-128.

569

570

571

572

573

574

575

576

577

578

579

580

581

582

583

584

585

586

587

588

589

590

591

592

593

594

Sanders TH, McMichael RW, Hendrix KW. 2000. Occurrence of Resveratrol in Edible Peanuts. Journal of agricultural and food chemistry 48(4):1243-1246

Song H, Guo ZL, Chen T, Sun J, Yang GF. 2018. Genome-wide identification of LRRcontaining sequences and the response of these sequences to nematode infection in Arachis duranensis. BMC Plant Biology 18:279.

Stark L, Nelke B, Hanbler G. 1997. Transfer of a grapevine stilbene synthase gene to rice (Oryza sativa L.). Plant Cell Reports 16:668-673.

Steuernagel B, Periyannan SK, Hernández-Pinzón I, Witek K, Rouse MN, Yu G, Hatta A, Ayliffe M, Bariana H, Jones JD Lagudah ES, Wulff BB. 2016. Rapid cloning of diseaseresistance genes in plants using mutagenesis and sequence capture. Nature Biotechnology. $34: 652-655$.

Takaoka MJ. 1940. Of the Phenolic Substances of White Hellebore (Veratrum grandiflorum Loes. fil.). Journal of the Faculty of Science Hokkaido Imperial University 3:1-16.

Tang K, Zhan JC, Yang HR, Huang WD. 2010. Changes of resveratrol and antioxidant enzymes during UV induced plant defense response in peanut seedlings. Journal of Plant Physiology 167:95-102.

Tian HY, Lv BS, Ding TT, Bai MY, Ding ZJ. 2017. Auxin-BR interaction regulates plant growth and development. Frontiers in Plant Science 8: 2256.

Tian LR, Liao BS, Huang JQ, Wang SY, Lei Y, Yan LY, Li D. 2008. Research progress on resveratrol in peanut. Chinese journal of oil crop sciences 30(4):522-528.

Wang H, Wang H, Shao H, Tang X. 2016. Recent advances in utilizing transcription factors to improve plant abiotic stress tolerance by transgenic technology. Frontiers in Plant Science $7: 67$.

Wang L, Hu W, Tie W, Ding Z, Ding X, Liu Y, Yan Y, Wu C, Peng M, Xu B, Jin Z. 2017. The MAPKKK and MAPKK gene families in banana: identification, phylogeny and expression during development, ripening and abiotic stress. Scientific Reports 7(1):1159. 
595

596

597

598

599

600

601

602

603

604

605

606

607

608

609

610

611

612

613

614

615

616

617

618

619

620

621

Wang W, Tang K, Yang HR, Wen PF, Zhang P, Wang HL. 2010. Distribution of resveratrol and stilbene synthase in young grape plants (Vitis vinifera L. cv. Cabernet Sauvignon) and the effect of UV-C on its accumulation. Plant Physiology and Biochemistry 48:142-152.

Wei H, Zhao H, Su T, Bausewein A, Greiner S, Harms K, Rausch T. 2017. Chicory R2R3-MYB transcription factors CiMYB5 and CiMYB3 regulate fructan 1-exohydrolase expression in response to abiotic stress and hormonal cues. Journal of Experimental Botany 68(15):43234338.

Xiong HC, Guo HJ, Xie YD, Zhao LS, Gu JY, Zhao SR, Li JH, Liu LX. 2017. RNAseq analysis reveals pathways and candidate genes associated with salinity tolerance in a spaceflightinduced wheat mutant. Scientific Reports 7(1):2731.

Yang S, Wang F, Wang F, Meng JJ, Li XG, Dong ST, Wan SB. 2013. Exogenous calcium alleviates photoinhibition of PSII by improving the xanthophyll cycle in peanut (Arachis hypogaea) leaves during heat stress under high irradiance. Plos one 8:e71214.

Yang Z, Wang C, Xue Y, Liu X, Chen S, Song C, Yang Y, Guo Y. 2019. Calcium-activated 143-3 proteins as a molecular switch in salt stress tolerance. Nature Commun 10(1):1199.

Zhang JL, Geng Y, Guo F, Li XG, Wan SB. 2020. Research progress on the mechanism of improving peanut yield by single-seed precision sowing. Journal of Integrative Agriculture doi: 10.1016/S2095-3119(19)62763-2.

Zhang Y, Wang PF, Xia H, Zhao CZ, Hou L, Li CS, Gao C, Zhao SZ, Wang XJ. 2016. Comparative transcriptome analysis of basal and zygote-located tip regions of peanut ovaries provides insight into the mechanism of light regulation in peanut embryo and pod development. BMC Genomics 17:606.

Zheng SG, Zhao SC, Li Z, Wang QG, Yao FY, Yang LQ, Pan JW, Liu W. 2015. Evaluating the effect of expressing a peanut resveratrol synthase gene in rice. PLos One 10(8):e0136013.

Zheng YP, Xin CY, Wang CB, Sun XS, Yang WQ, Wan SB, Zheng YM, Feng H, Chen DX, Sun XW, Wu ZF. 2013. Effects of phosphorus fertilizer on root morphology, physiological characteristics and yield in peanut (Arachis hypogaea). Chinese Journal of Plant Ecology

Peer) reviewing PDF | (2020:04:48461:5:0:NEW 23 Nov 2020) 
623 Zhu JK. 2016. Abiotic stress signaling and responses in plants. Cell 167:313-324.

\section{Figure Legends}

625 Fig. 1 Phenotypical comparisons of peanut in SS and DS sowing treatment. (a) The phenotypes, 626 (b) absorbing area of the root in SS and DS, and (c) Dry weight of root in SS and DS.

627 Fig. 2 Scatter plot of DEGs in leaf and root between SS and DS treatments.

628 Fig. 3 Differentially expressed genes analysis in roots and leaves. (a) Venn diagram 629 demonstrated the common and specific differentially expressed genes (DEGs), (b) Heat map 630 demonstrated the expression profile DEGs.

631 Fig. 4 qRT-PCR verification of randomly selected genes. Heatmaps represent the expression of 632 the 22 DEGs in (a) leaves and (b) roots between SS and DS treatments. For each heatmap, the 633 FPKM values increased from green to red. The correlation of the fold change in (c) leaves and (d) 634 roots analyzed by RNA-Seq (x-axis) with data obtained using qRT-PCR (y-axis).

635 Fig. 5 GO annotation among DEGs identified in the leaf of SS treatment compared with DS 636 treatment and root from the two different treatments. The GO ID was listed alongside the textual 637 annotations.

638 Fig. 6 Co-expression of DEGs between SS and DS treatments. (a) Hierarchical of 10,000 genes 639 based on topological overlap and visualization of gene modules with assigned colors. Branches 640 in the hierarchical clustering dendrograms correspond to modules. Color-coded module 641 membership is displayed in the colored bars below and to the right of the dendrograms. (b) The 642 network of co-expressed modules. The colored clustering bars directly correspond to the module 643 (color) designation for the clusters of genes.

644 Fig. 7 Determination of resveratrol contents according to the method detailed by Tang (Tang et $645 a l ., 2010$ ) in leaf and root between SS and DS treatments. 
649 Fig. S1 The SNP variants were summarized for each sample. A-G: The amount of A-G variant 650 type; C-T: The amount of C-T variant type; Transition: The amount of A-G and C-T variant type; 651 A-C: The amount of A-C variant type; A-T: The amount of A-T variant type; C-G: The amount 652 of C-G variant type; G-T: The amount of G-T variant type; Transversion: The amount of A-C, 653 A-T, C-G, and G-T variant type.

654 Fig. S2 The distributions of INDEL location for each sample were analyzed. Up2k means 655 upstream 2,000 bp area of a gene. Down2k means downstream 2,000 bp area of a gene.

656 Fig. S3 Based on the SNP, INDEL, and gene expression result, Circos were used to perform the 657 analysis.

658 Fig. S4 Pathway functional enrichment of DEGs. X axis represents the enrichment factor. Y axis 659 represents the pathway name. The color indicates the q-value (high: white, low: blue); the lower 660 q-value indicates the more significant enrichment. Point size indicates DEG number (The bigger 661 662 dots refer to a larger amount). Rich Factor refers to the value of enrichment factor, which is the quotient of foreground value (the number of DEGs) and background value (total Gene amount).

663 Table S1 Variety of the activities of SOD, POD, CAT, and TTC in SS and DS sowing treatments, 664 respectively. The data presented are the mean values $\pm \mathrm{SD}$ of three individual experiments. 


\section{Figure 1}

Phenotypical comparisons of peanut in SS and DS sowing treatment.

(a) The phenotypes, (b) absorbing area of root in SS and DS, and (c) Dry weight of root in SS and DS. Fig. 2 Scatter plot of DEGs in leaf and root between SS and DS treatments.
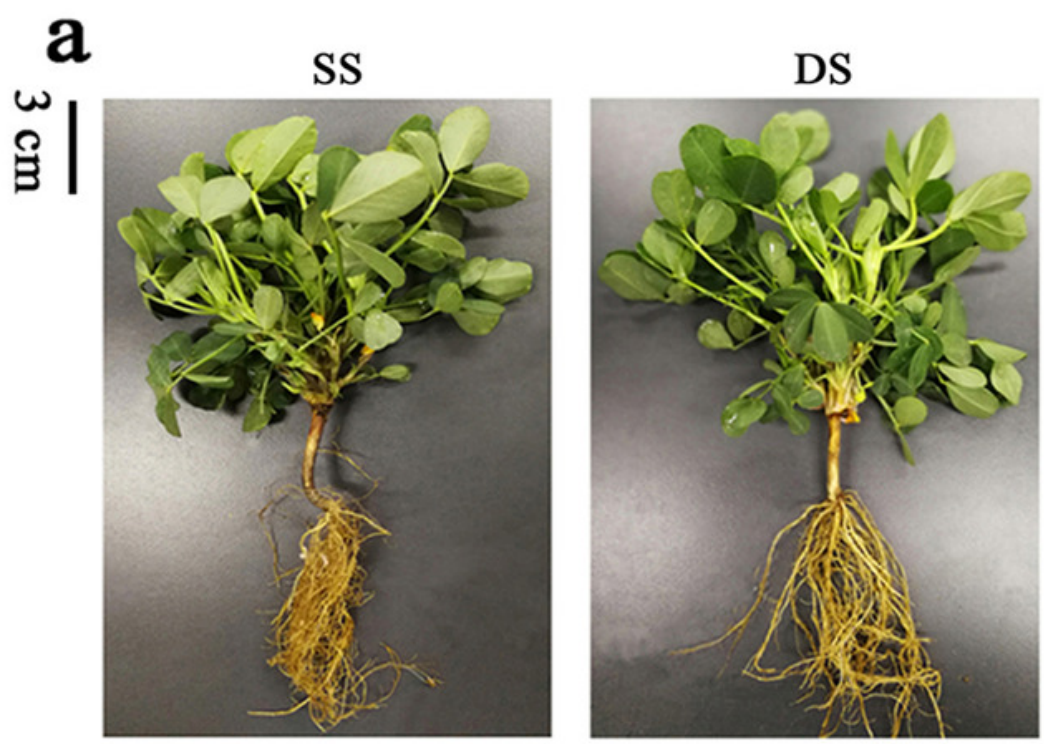

b

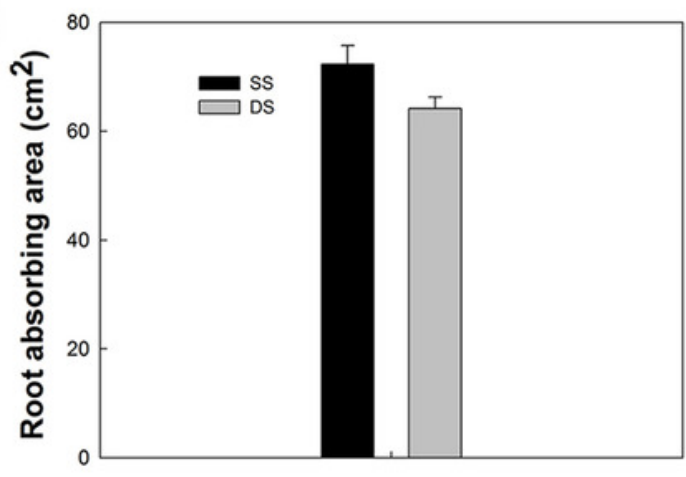

C

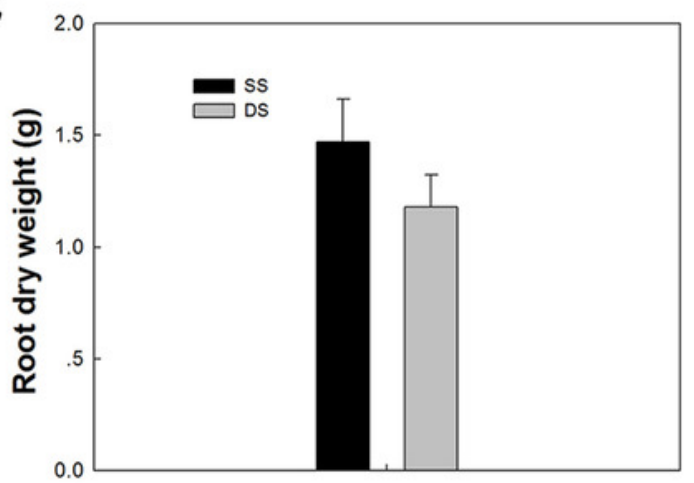


Figure 2

Fig. 2 Scatter plot of DEGs in leaf and root between SS and DS treatments.
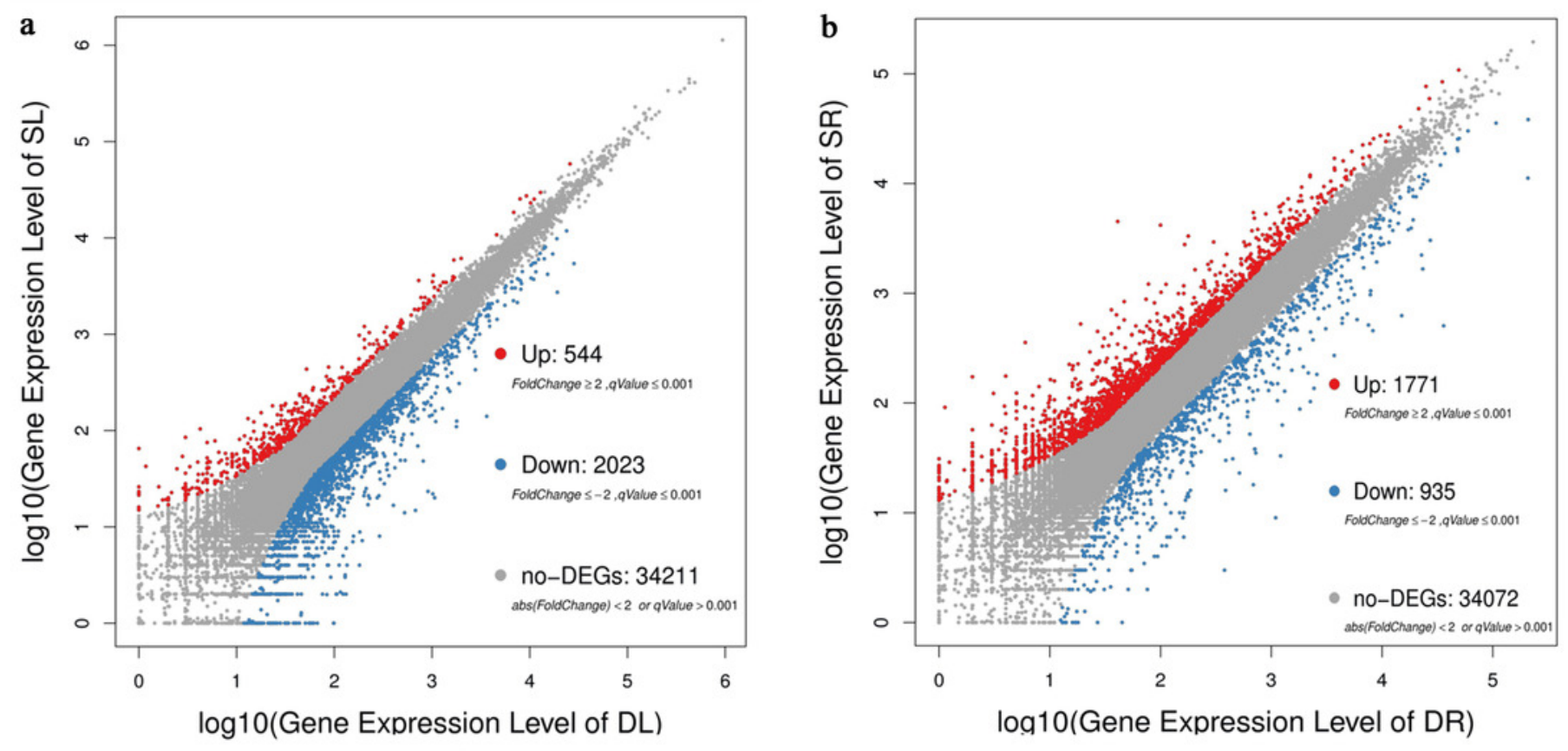

c

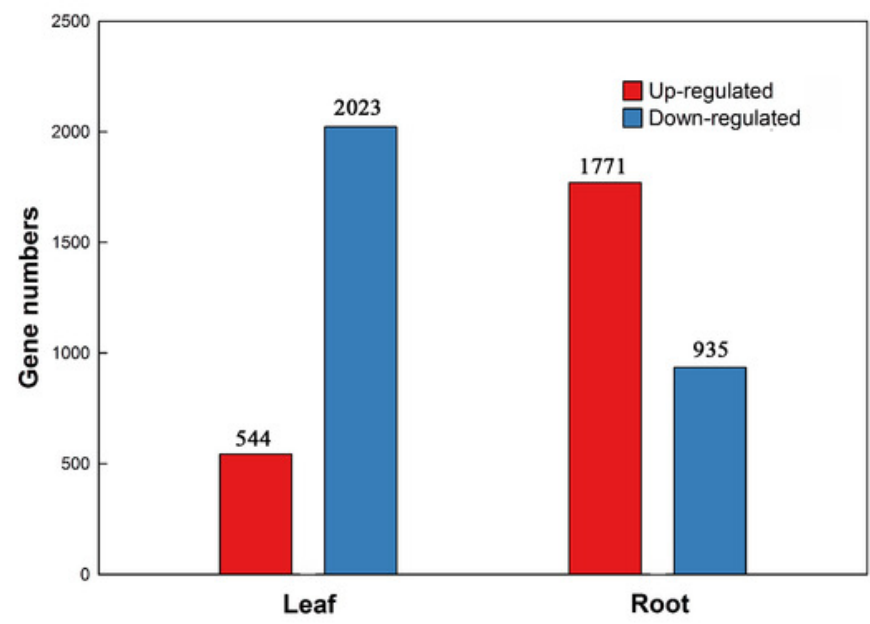




\section{Figure 3}

Fig. 3 Differentially expressed genes analysis in roots and leaves.

(a) Venn diagram demonstrated the common and specific differentially expressed genes (DEGs), (b) Heat map demonstrated the expression profile DEGs.

a

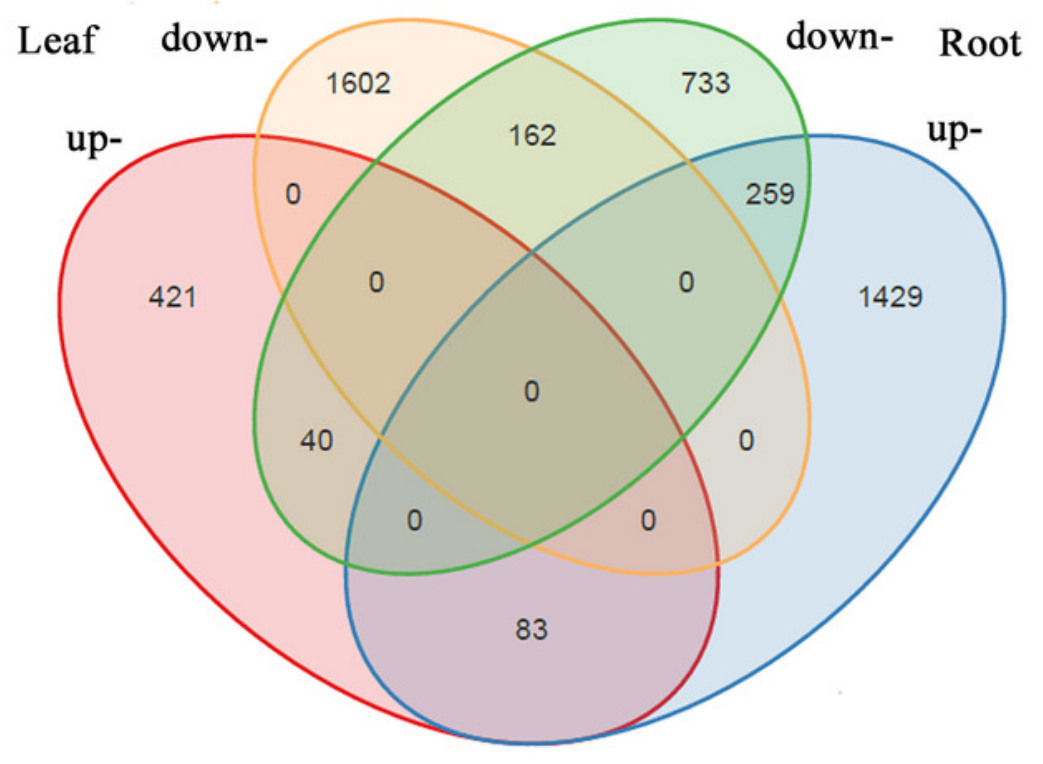

b

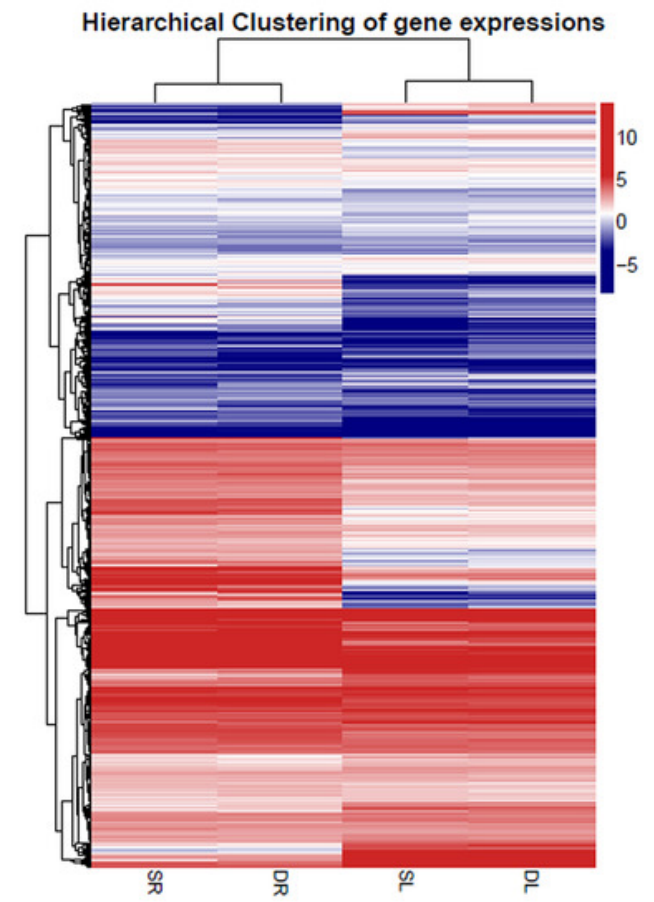


Figure 4

Fig. 4 qRT-PCR verification of randomly selected genes.

Heatmaps represent the expression of the 22 DEGs in (a) leaves and (b) roots between SS and DS treatments. For each heatmap, the FPKM values increased from green to red. The correlation of the fold change in (c) leaves and (d) roots analyzed by RNA-Seq (x-axis) with data obtained using qRT-PCR (y-axis). 
a qRT-PCR RNA-seq

b qRT-PCR RNA-seq
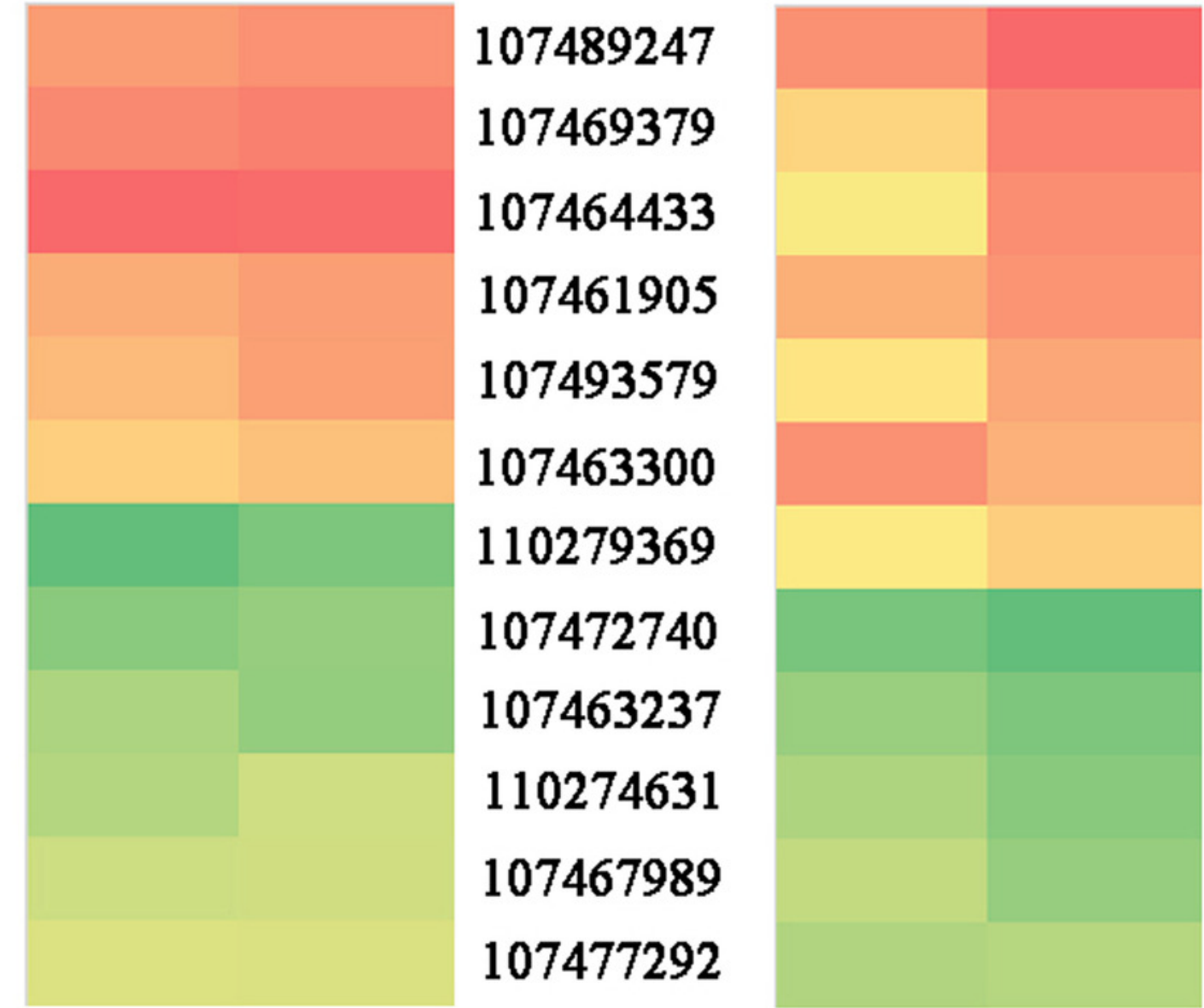

107476234

107482875

107474885

107494567

107465287

110273860

107479532

107460667

107488919

107489790

107492649

107464364

c

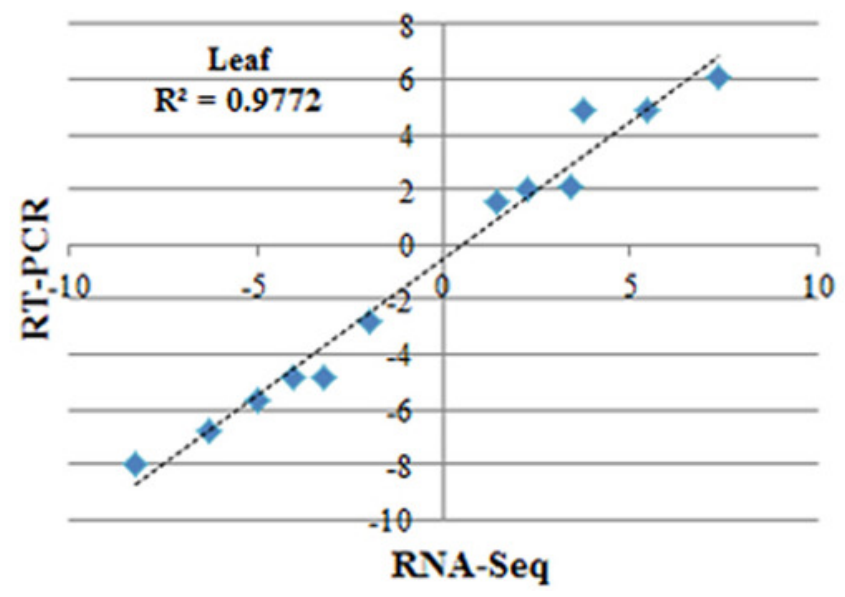

d

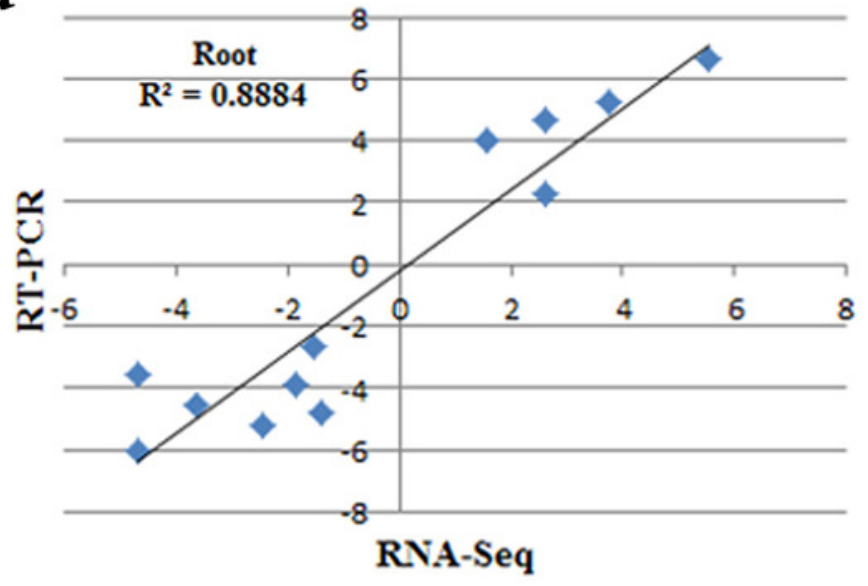


Figure 5

Fig. 5 GO annotation among DEGs identified in the leaf of SS treatment compared with DS treatment and root from the two different treatments.

The GO ID was listed alongside the textual annotations.

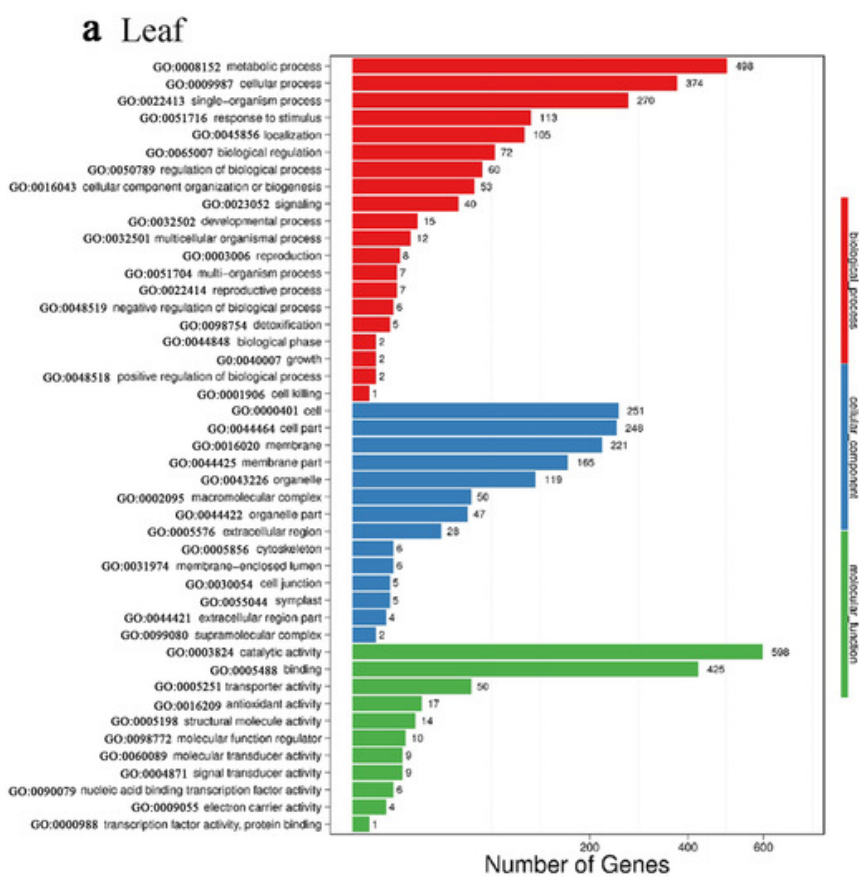

b Root

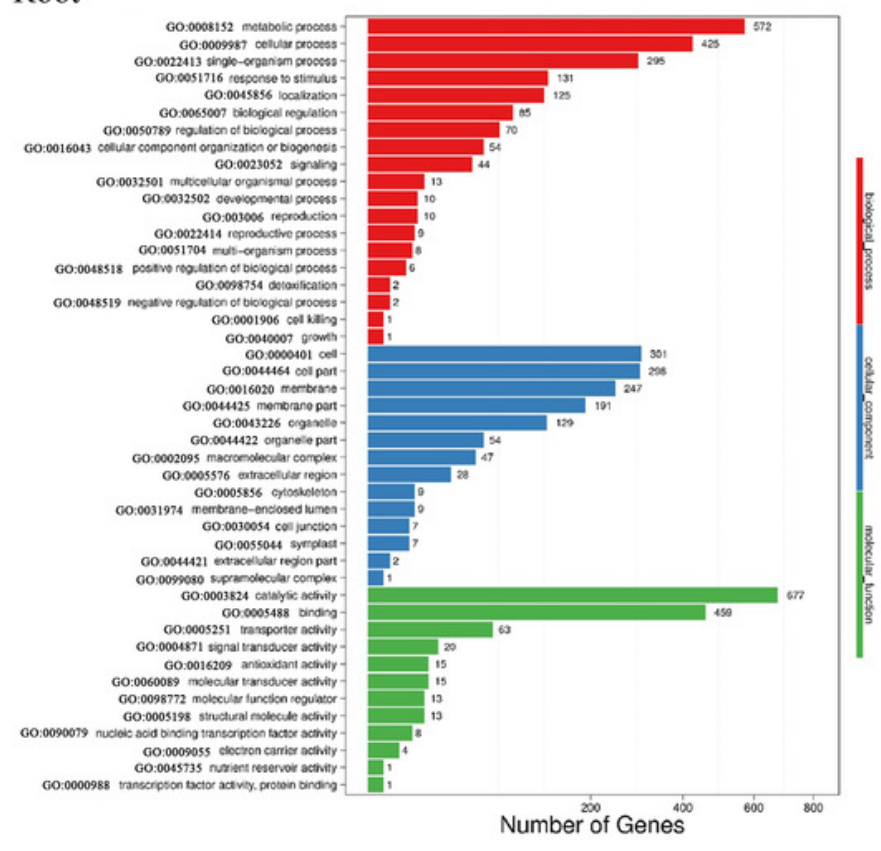




\section{Figure 6}

Fig. 6 Co-expression of DEGs between SS and DS treatments.

(a) Hierarchical of 10,000 genes based on topological overlap and visualization of gene modules with assigned colors. Branches in the hierarchical clustering dendrograms correspond to modules. Color-coded module membership is displayed in the colored bars below and to the right of the dendrograms. (b) Network of co-expressed modules. The colored clustering bars directly correspond to the module (color) designation for the clusters of genes.

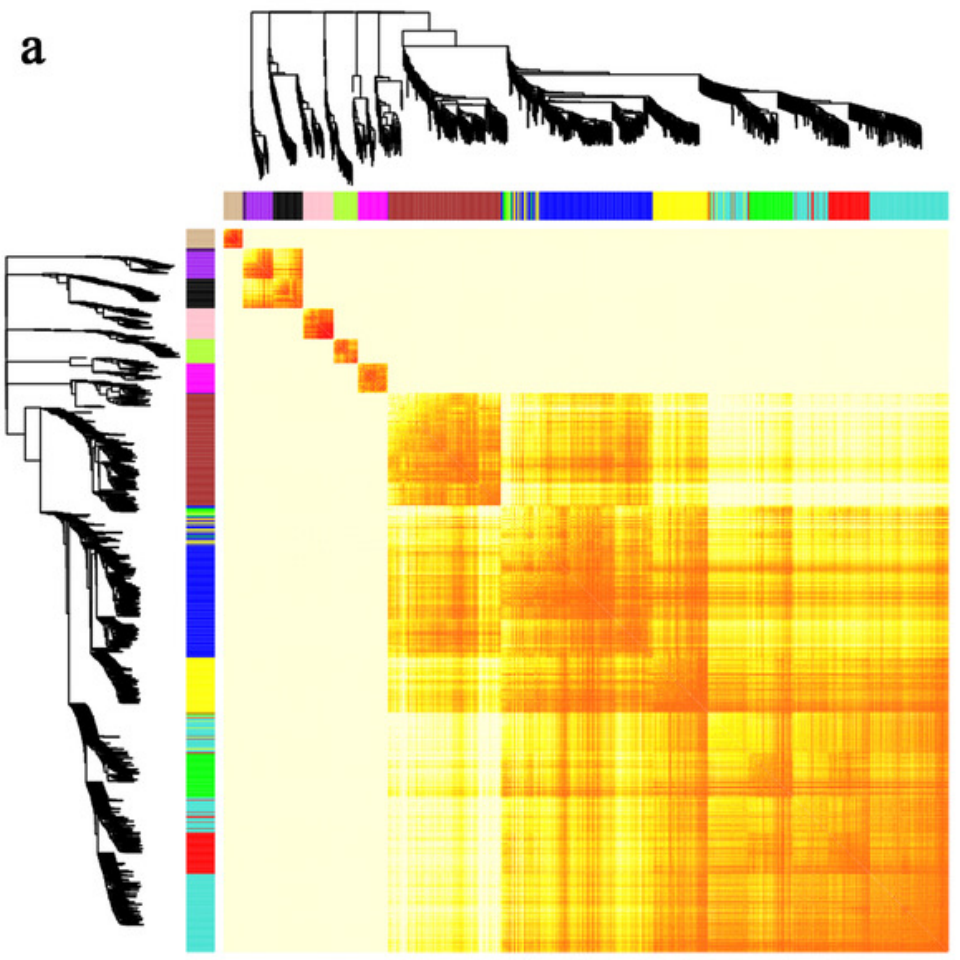

b

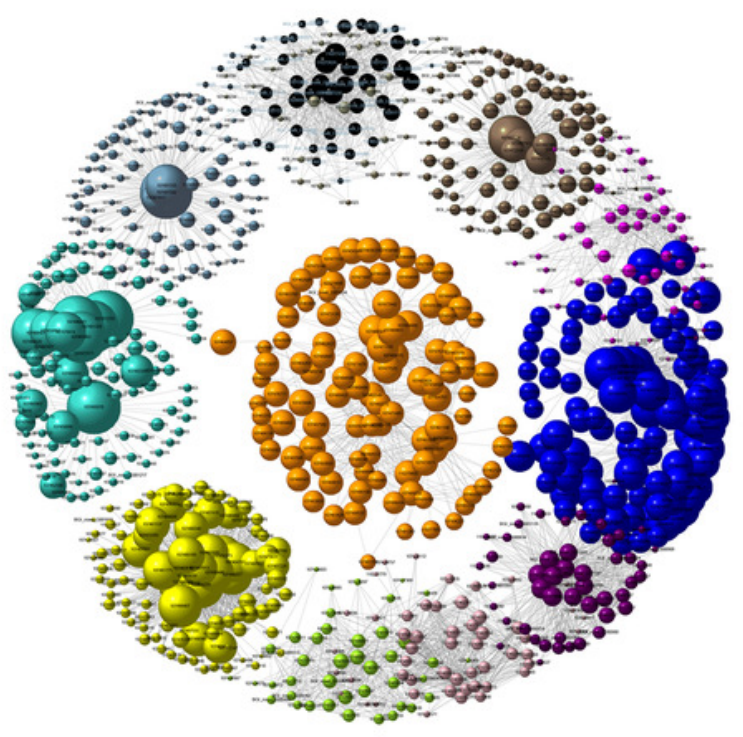


Figure 7

Fig. 7 Determination of resveratrol contents according to the method detailed by Tang (Tang et al., 2010) in leaf and root between SS and DS treatments.

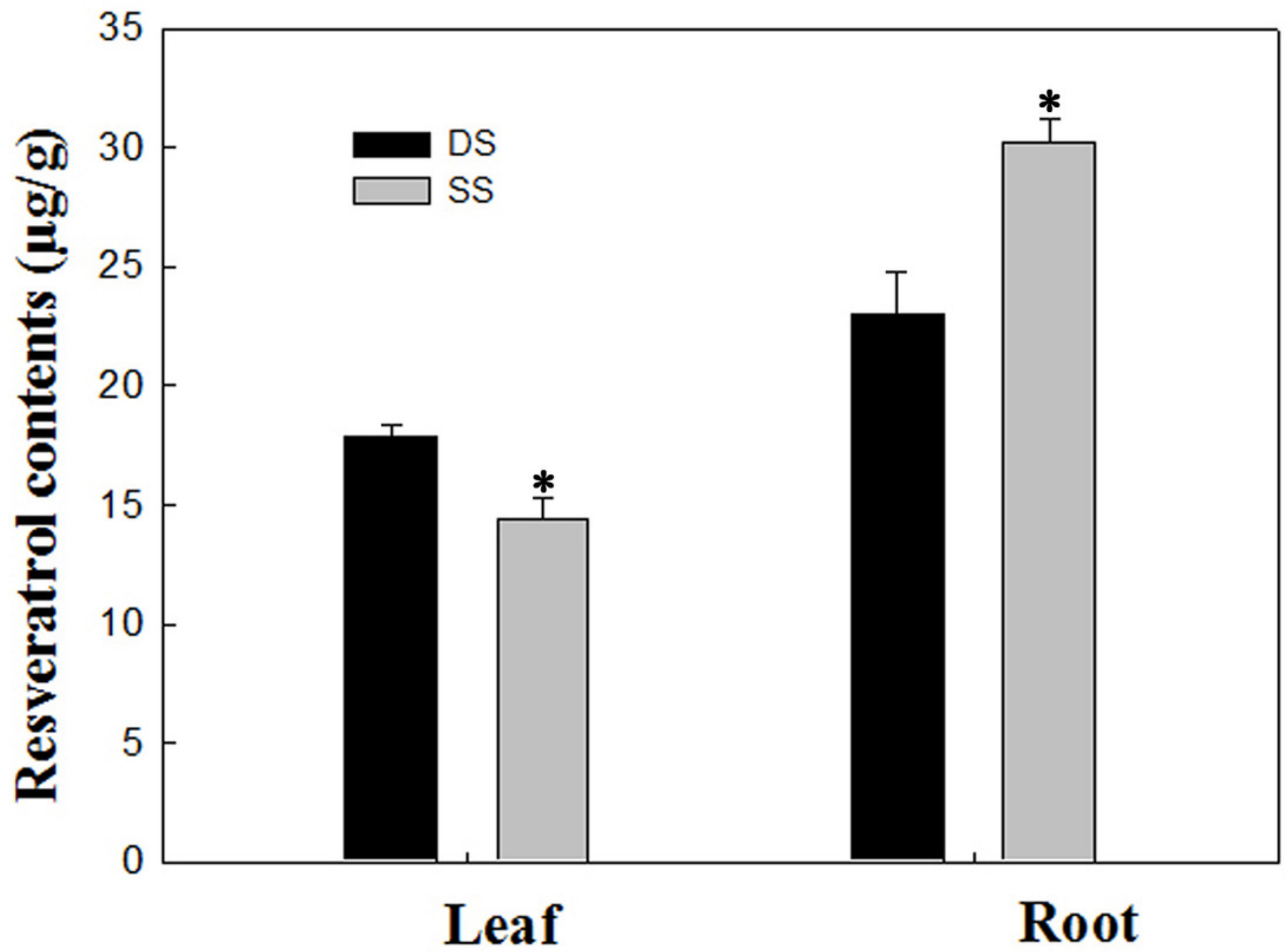




\section{Table $\mathbf{1}$ (on next page)}

Table 1 Summary of read numbers from leaves and roots.

SL: Leaf in single-seed (SS) precision sowing; DL: Leaf in double-seed (DS) sowing; SR: Root in single-seed (SS) precision sowing; DR: Root in double-seed (DS) sowing. 
Table 1 Summary of read numbers from leaves and roots. SL: Leaf in single-seed (SS) precision sowing; DL: Leaf in double-seed (DS) sowing; SR: Root in single-seed (SS) precision sowing; DR: Root in double-seed (DS) sowing.

4

$$
\text { Samples }
$$

Total clean reads

Total Mapping Ratio

Unique match Ratio

Unmapped reads

Novel transcript number

SL1 SL2

$\begin{array}{lll}45,001,686 & 44,475,256 & 44,262\end{array}$

$75.27 \%$

$55.15 \%$

$24.73 \%$

13,152 $\begin{array}{lllll}45,001,686 & 44,475,256 & 44,262,552 & 42,022,054 & 42,2 \\ 75.27 \% & 74.87 \% & 74.88 \% & 78.26 \% & 80.5\end{array}$

$53.51 \% \quad 54.34 \%$

$25.13 \% \quad 25.12 \%$

$78.26 \%$
$49.41 \%$

\begin{tabular}{lll}
$53.51 \%$ & $54.34 \%$ & $49.41 \%$ \\
$25.13 \%$ & $25.12 \%$ & $21.74 \%$ \\
12,615 & 13,149 & 13,218 \\
\hline
\end{tabular}

5
6
7
8
9
10
11
12
13
14
15
16
17
18
19
20




\section{Table 2 (on next page)}

Table 2 Analysis of resveratrol synthesis related genes in leaves and roots.

The ratio was obtained from the transcriptome results. SL: Leaf in single-seed (SS) precision sowing; DL: Leaf in double-seed (DS) sowing; SR: Root in single-seed (SS) precision sowing; DR: Root in double-seed (DS) sowing. 
Table 2 Analysis of resveratrol synthesis related genes in leaves and roots. The ratio was obtained from the transcriptome results. SL: Leaf in single-seed (SS) precision sowing; DL: Leaf in double-seed (DS) sowing; SR: Root in single-seed (SS) precision sowing; DR: Root in double-seed (DS) sowing.

\begin{tabular}{cccc}
\hline & Annotation & $\log _{2}(\mathrm{SL} / \mathrm{DL})$ & Gene expression change \\
& resveratrol synthase & -1.43 & Down-regulated \\
& resveratrol synthase & -1.92 & Down-regulated \\
& resveratrol synthase & -2.03 & Down-regulated \\
& resveratrol synthase & -2.31 & Down-regulated \\
Leaf & resveratrol synthase & -2.66 & Down-regulated \\
& resveratrol synthase & -2.77 & Down-regulated \\
& resveratrol synthase & -3.36 & Down-regulated \\
& resveratrol synthase & -6.66 & Down-regulated \\
& stilbene synthase & -1.18 & Down-regulated \\
& stilbene synthase & -1.38 & Down-regulated \\
\hline Annotation & $\log _{2}(\mathrm{SR} / \mathrm{DR})$ & Gene expression change \\
& resveratrol synthase & 2.67 & Up-regulated \\
resveratrol synthase & 2.59 & Up-regulated \\
resveratrol synthase & 2.37 & Up-regulated \\
& resveratrol synthase & 2.27 & Up-regulated \\
resveratrol synthase & 2.24 & Up-regulated \\
resveratrol synthase & 2.13 & Up-regulated \\
resveratrol synthase & 2.12 & Up-regulated \\
resveratrol synthase & 2.05 & Up-regulated \\
resveratrol synthase & 1.86 & Up-regulated \\
resveratrol synthase & 1.80 & Up-regulated \\
resveratrol synthase & 1.68 & Up-regulated \\
resveratrol synthase & 1.67 & Up-regulated \\
resveratrol synthase & 1.61 & Up-regulated \\
\hline
\end{tabular}




\begin{tabular}{ccc}
\cline { 2 - 3 } resveratrol synthase & 1.56 & Up-regulated \\
resveratrol synthase & 1.55 & Up-regulated \\
resveratrol synthase & 1.47 & Up-regulated \\
resveratrol synthase & 1.34 & Up-regulated \\
resveratrol synthase & 1.27 & Up-regulated \\
stilbene synthase & 2.47 & Up-regulated \\
stilbene synthase & 2.08 & Up-regulated \\
stilbene synthase & 1.19 & Up-regulated \\
\hline
\end{tabular}




\section{Table 3 (on next page)}

Table 3 List of putative candidate genes for high yields in SS sowing treatment.

These candidate genes were up-regulated in SR vs DR comparison groups with the corrected $p$ value $<0.05$ and are here classified according to predicted gene function. The values in the columns are the log2 Fold Change values for the SR vs DR comparison groups obtained from the transcriptome results. SR: Root in single-seed (SS) precision sowing; DR: Root in double-seed (DS) sowing. 
1 Table 3 List of putative candidate genes for high yields in SS sowing treatment. These candidate

2 genes were up-regulated in SR vs DR comparison groups with the corrected $p$ value $<0.05$ and

3 are here classified according to predicted gene function. The values in the columns are the $\log 2$

4 Fold Change values for the SR vs DR comparison groups obtained from the transcriptome results.

5 SR: Root in single-seed (SS) precision sowing; DR: Root in double-seed (DS) sowing.

\begin{tabular}{|c|c|c|}
\hline Gene_id & Gene description & SR vs DR \\
\hline \multicolumn{3}{|l|}{ Oxidation-reduction } \\
\hline 107460187 & L-ascorbate oxidase homolog & 1.4 \\
\hline 110279516 & L-ascorbate oxidase & 1.4 \\
\hline 107482474 & L-ascorbate oxidase & 1.2 \\
\hline 107480398 & L-ascorbate oxidase & 1.1 \\
\hline 107471312 & glutaredoxin & 2.8 \\
\hline 107475918 & glutaredoxin 3 & 2.4 \\
\hline 107494759 & glutaredoxin-C1-like & 1.5 \\
\hline 107461150 & glutaredoxin 3 & 1.1 \\
\hline 107475477 & cytochrome P450 83B1-like & 2.2 \\
\hline 107491506 & cytochrome P450 71A1-like & 2.1 \\
\hline 107475482 & cytochrome P450 83B1-like & 2.0 \\
\hline 107475708 & cytochrome P450 83B1-like & 3.5 \\
\hline 107478833 & cytochrome P450 84A1-like & 1.1 \\
\hline 107475710 & cytochrome P450 71A1-like & 1.2 \\
\hline 107487896 & cytochrome P450 71D8-like & 1.2 \\
\hline 107492914 & cytochrome P450 93A3 & 1.3 \\
\hline \multicolumn{3}{|l|}{ Hormone related } \\
\hline 107491535 & Protein brassinosteroid & 1.7 \\
\hline 107486286 & gibberellin-regulated protein & 2.4 \\
\hline BGI_novel_G000490 & gibberellin receptor GID1 & 2.0 \\
\hline 107495262 & gibberellin receptor GID1 & 2.6 \\
\hline 107476131 & gibberellin receptor GID1 & 1.9 \\
\hline 107464975 & gibberellin 2-beta-dioxygenase 2-like & 1.7 \\
\hline 107477445 & DELLA protein & 1.7 \\
\hline 107491113 & DELLA protein & 1.1 \\
\hline 107476118 & auxin responsive GH3 gene family & 1.9 \\
\hline 107465048 & auxin-responsive protein IAA & 1.8 \\
\hline 107492413 & auxin response factor & 1.9 \\
\hline 107463844 & auxin-responsive protein IAA & 1.4 \\
\hline 107462192 & auxin responsive GH3 gene family & 1.8 \\
\hline 107459619 & auxin-responsive protein IAA & 1.5 \\
\hline 107478268 & auxin responsive GH3 gene family & 1.0 \\
\hline 107464096 & auxin-responsive protein IAA & 1.2 \\
\hline
\end{tabular}




\begin{tabular}{|c|c|c|}
\hline 107495645 & lipoxygenase & 2.4 \\
\hline 107464479 & linoleate 9S-lipoxygenase & 1.1 \\
\hline \multicolumn{3}{|c|}{ Transcription factor and signaling regulation } \\
\hline 107491013 & MADS-box transcription factor & 3.0 \\
\hline 107476111 & MADS-box transcription factor & 5.7 \\
\hline 107458618 & zinc finger protein CONSTANS-LIKE 4-like & 2.8 \\
\hline 107492404 & Zinc finger protein & 2.4 \\
\hline 110279598 & zinc finger protein 3-like & 2.3 \\
\hline 107493757 & zinc finger protein-like protein & 1.8 \\
\hline 107481180 & Zinc finger protein 6 & 1.5 \\
\hline 107460097 & $\mathrm{C} 2 \mathrm{H} 2-$ like zinc finger protein & 1.3 \\
\hline 107483222 & MYB family transcription factor & 4.8 \\
\hline 107461398 & MYB86 Transcription factor & 1.5 \\
\hline 107475194 & MYB transcription factor MYB51 & 1.2 \\
\hline 107491849 & R2R3 MYB protein 2 & 1.2 \\
\hline 107459300 & WRKY transcription factor 70 & 2.4 \\
\hline 107472118 & WRKY transcription factor & 1.8 \\
\hline 107472768 & WRKY transcription factor 22-like & 1.5 \\
\hline 107481590 & WRKY transcription factor 14 & 1.3 \\
\hline 107463444 & ERF114-like & 2.4 \\
\hline 107488356 & ERF022 & 1.6 \\
\hline 107464842 & ERF13 & 1.5 \\
\hline 107471058 & ERF WRI1 & 1.0 \\
\hline 107486141 & ERF 1-like & 1.0 \\
\hline BGI_novel_G002321 & bZIP & 7.3 \\
\hline 107487341 & bZIP & 1.5 \\
\hline 107466101 & Calcium-binding protein CML & 1.4 \\
\hline 107491924 & Calcium-binding protein KIC-like & 1.3 \\
\hline 107460313 & Calcium-binding protein CML & 1.2 \\
\hline 107479434 & MAPKKK & 1.5 \\
\hline \multicolumn{3}{|l|}{ Disease resistance } \\
\hline 107468687 & pathogenesis-related protein 1 & 2.2 \\
\hline 107468499 & pathogenesis-related protein 1 & 1.8 \\
\hline 107468493 & pathogenesis-related protein 1 & 1.4 \\
\hline 107474846 & pathogenesis-related protein 1 & 1.0 \\
\hline 107460041 & 4-coumarate--CoA ligase & 4.2 \\
\hline 107472893 & 4-coumarate--CoA ligase & 3.1 \\
\hline 107480465 & 4-coumarate--CoA ligase & 2.6 \\
\hline 107458085 & 4-coumarate--CoA ligase & 2.0 \\
\hline 107466660 & 4-coumarate--CoA ligase-like 5 & 1.7 \\
\hline
\end{tabular}


6

7

8

9 


\section{Table 4 (on next page)}

Table 4 Pod per plant at maturity stage of peanut under different planting patterns 
1 Table 4 Pod per plant at maturity stage of peanut under different planting patterns

\begin{tabular}{llllll}
\hline Treatment & Pods number per plant & Full pods number per plant & Double kernel number per plant & Pod weight per plant (g) & Economic coefficient \\
\hline SS & $39.1 \mathrm{a}$ & $22.6 \mathrm{a}$ & $24.4 \mathrm{a}$ & $51.7 \mathrm{a}$ & $0.49 \mathrm{a}$ \\
DS & $31.8 \mathrm{~b}$ & $16.3 \mathrm{~b}$ & $16.9 \mathrm{~b}$ & $39.3 \mathrm{~b}$ & $0.45 \mathrm{~b}$ \\
\hline
\end{tabular}

2 